\title{
SPECTROSCOPIC DIAGNOSTICS FOR N III ION
}

\author{
B.N. DWIVEDI, ANITA MOHAN and A.K. GUPTA \\ Department of Applied Physics, Institute of Technology \\ Banaras Hindu University, Varanasi 221005 . India
}

September 19, 1996

\section{Extended Abstract}

Spectroscopic diagnostics for the N III ion have been studied using an atomic model comprising of the first eleven energy levels. We have taken account of the various populating and depopulating mechanisms to solve the detailed balance equations to determine the level populations as a function of electron density and temperature. We then studied the line emissivity ratios as tools for density and temperature diagnostics. Density-sensitive and temperature-sensitive line emissivity ratios have been presented and their diagnostic applications to the solar atmosphere have been discussed with the help of available observations.

The line ratios from the ${ }^{2} \mathrm{P}^{0}-{ }^{4} \mathrm{P}$ multiplet namely, $\lambda 1753.97 / \lambda 1752.05, \lambda 1748.64 / \lambda 1752.05$, $\lambda 1748.64 / \lambda 1749.67, \lambda 1753.97 / \lambda 1749.67, \lambda 1752.05 / \lambda 1749.67$ and $\lambda 1746.72 / \lambda 1748.64$ are found to be good tools for density measurements in the emitting source. These line ratios are density-sensitive in the density range $10^{8}-10^{11} \mathrm{~cm}^{-3}$ and ideal for density measurements in the chromosphere corona transition region and other astrophysical sources as they are insensitive to temperature variations. In addition, we have also carried out an extensive analysis of several line pairs in the SUMER spectral range (500-1600 $\AA$ ) from the N III ion at the temperature of its peak ionic concentration ( $\mathrm{T} \sim 8 \times 10^{4} \mathrm{~K}$ ), e.g., $\lambda 772.38 / \lambda 763.33$, $\lambda 772.38 / \lambda 989.80, \lambda 771.92 / \lambda 685.81, \lambda 772.38 / \lambda 764.35, \lambda 772.38 / \lambda 685.81, \lambda 772.38 / \lambda 991.58$ and $\lambda 771.92 / \lambda 991.58$ which are found to be good density indicators in the density range $10^{8}-10^{11} \mathrm{~cm}^{-3}$. Our study of the temperature-sensitive line ratio $\lambda 991 / \lambda 686$ indicated $\mathrm{a}$ lower temperature of line formation of $\mathrm{N}$ III ion $\left(\mathrm{T}_{e} \sim 4 \times 10^{4} \mathrm{~K}\right)$ than the temperature of its maximum fractional abundance $\left(\mathrm{T}_{\max } \sim 8 \times 10^{4} \mathrm{~K}\right)$. Temperature-sensitive theoretical line ratios for resolved line components have also been reported. Observations from the Solar Ultraviolet Measurements of Emitted Radiation (SUMER) experiment aboard the Solar and Heliospheric Observatory (SOHO) mission will provide a rich source of data at greater spectral, spatial and temporal resolution than currently available to study the density and temperature structure in the chromosphere-corona transition region and fine structures in it, making use of the present investigation. 